\title{
Fine-tuning of Distributed Network Covert Channels Parameters and Their Impact on Undetectability
}

\author{
Krzysztof Cabaj \\ Warsaw University of Technology \\ Warsaw, Poland \\ kcabaj@ii.pw.edu.pl \\ Piotr Nowakowski \\ Warsaw University of Technology \\ Warsaw, Poland \\ p.nowakowski@tele.pw.edu.pl
}

\author{
Wojciech Mazurczyk \\ Warsaw University of Technology \\ Warsaw, Poland \\ wmazurczyk@tele.pw.edu.pl \\ Piotr Żórawski \\ Warsaw University of Technology \\ Warsaw, Poland \\ p.zorawski@tele.pw.edu.pl
}

\begin{abstract}
Currently the usage of various information hiding techniques for nefarious purposes becomes a major issue. Especially when the attackers, in order to stay under the radar, utilize increasingly sophisticated ways to conceal data. One of such advanced techniques is the use of distributed network covert channels (DNCC) where the secrets are spread among a number of available covert channels and transmitted in parallel. Taking above into consideration, in this paper we investigate how the data hiding techniques forming a DNCC can be configured to achieve an improved undetectability and how this impacts the DNCC performance and its detection susceptibility. Based on the presented results it can be concluded that if the utilized steganographic techniques forming a DNCC are used in a balanced manner then this may pose difficulties from the detection perspective although this typically also means significant limitation of the overall secret data rate.
\end{abstract}

\section{CCS CONCEPTS}

- Security and privacy $\rightarrow$ Network security; Distributed systems security; Information flow control; Pseudonymity, anonymity and untraceability.

\section{KEYWORDS}

network covert channels, network steganography, information hiding, distributed network covert channel

\section{ACM Reference Format:}

Krzysztof Cabaj, Wojciech Mazurczyk, Piotr Nowakowski, and Piotr Żórawski. 2019. Fine-tuning of Distributed Network Covert Channels Parameters and Their Impact on Undetectability. In Proceedings of the 14th International Conference on Availability, Reliability and Security (ARES 2019) (ARES '19), August 26-29, 2019, Canterbury, United Kingdom. ACM, New York, NY, USA, 8 pages. https://doi.org/10.1145/3339252.3341489

Permission to make digital or hard copies of part or all of this work for personal or classroom use is granted without fee provided that copies are not made or distributed for profit or commercial advantage and that copies bear this notice and the full citation on the first page. Copyrights for third-party components of this work must be honored For all other uses, contact the owner/author(s).

ARES '19, August 26-29, 2019, Canterbury, United Kingdom

(C) 2019 Copyright held by the owner/author(s).

ACM ISBN 978-1-4503-7164-3/19/08.

https://doi.org/10.1145/3339252.3341489

\section{INTRODUCTION}

In the last few years, various information hiding techniques have been used by the cybercriminals in communication networks for nefarious purposes [16], [9]. Among the variety of potential methods so called network covert channels [15], [10], [4] are increasingly used by the attackers to cloak their malicious activities e.g. to invisibly communicate with the Command \& Control (C\&C) servers, exfiltrate confidential/sensitive data, or download further malware modules [2], [7]. It must be also noted that it is expected that in the future this trend is likely to continue and we will witness a proliferation of sophisticated covert channel techniques in malware and in network attacks [9], [2], [13] which will be a real challenge for the digital forensics and security community [6].

Moreover, it is foreseen that even more complex forms of the network covert channels will be utilized in response to the increasingly efficient and effective information hiding countermeasures [5]. Therefore, it is vital to explore other "constructions" of data hiding techniques especially those that enable to achieve improved undetectability and/or covert channel bandwidth. One example are information hiding-based botnets which may offer completely covert exchange of the malicious content [1], [12], [11].

Another example of the sophisticated form of covert channels are distributed network covert channels (DNCCs) [8], [3]. DNCCs are defined as the network covert channels that spread the secret data among many flows/protocols/hosts or use multiple data hiding methods within the same flow or within PDUs in order to provide hidden data exchange. In contrast, the typical (undistributed) network covert channels are storage or a timing channels that in order to embed secret data utilize PDUs of a single flow or protocol.

As mentioned DNCCs are nowadays raising increased attention among security community as on one hand they can improve the overall stealthiness and bandwidth of the hidden communication but on the other hand they can be used to bypass existing defensive solutions. However, it must be noted that so far in the literature no systematic analyses of the DNCCs both from the properties perspective as well as from the detection angle have been presented.

Our previous works contributed to the investigation of the distributed network covert channels. In [8] we extended a network information hiding patterns concept so it is able to incorporate DNCCs and, additionally, we introduced their novel taxonomy. 


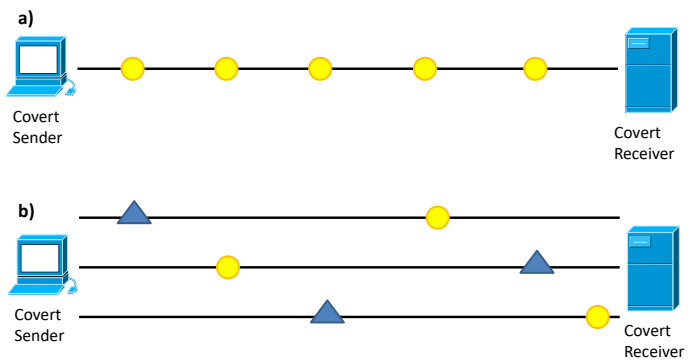

Figure 1: Comparison of the typical (a) and distributed (b) network covert channel.

Moreover, in [3] we have evaluated whether data mining techniques can be suitable for the distributed network covert channels detection. We have considered the typical state-of-the-art network covert channel which is based on the IPv4 TTL field values modulation and additionally the secret data is distributed among several data flows. The initial results that we obtained were promising and thus we concluded that such an approach should be further investigated.

When compared with the previous works mentioned above in this paper our main novel contribution is that we perform a thorough investigation of the DNCCs capabilities, and in particular, we provide more insights on how DNCCs should be constructed and used in order to achieve increased undetectability. Moreover, we develop an exemplary proof-of-concept implementation of DNCC and perform its experimental evaluation.

The rest of this paper is structured as follows. Section 2 describes the basic information on distributed network covert channels and details three steganographic methods which we used for the DNCC creation in this paper. Next, in Section 3 experimental methodology and test-bed is outlined followed by the obtained results. Finally, Section 4 concludes our work and outlines potential future steps.

\section{DISTRIBUTED NETWORK COVERT CHANNELS}

The main difference between the DNCC and typical network covert channel is illustrated in Fig. 1. As we can observe DNCC is significantly more complex information hiding system where the data can be distributed across many available data hiding techniques operating within the same connection or within the number of available connections between the covert sender and the covert receiver. As already mentioned in our previous works [8], [3] the DNCC relies on utilization of several data hiding techniques which create many network covert channels that can be used simultaneously (or alternatively interleaved).

It must be also noted that in [14] authors introduce a classification of network hiding techniques into so-called patterns where information hiding patterns provide an abstract description of a hiding technique and are categorized in a hierarchy. What must be emphasized is that each hiding pattern is a unified and generic description of a particular hiding method. From this perspective, in Fig. 1(b) each symbol (a circle or a triangle) represents a certain change in the hidden data carrier used for a specific method (e.g., a packet, a flow, etc.) related to the embedding of secret data. In the remaining of this paper we call such an event as information hiding pattern instance (IHPI).

It must be noted that in our previous work [3] we determined that for the data mining-based detection it is possible to discover the DNCC, especially, if it is constructed in a naive manner. This means that if the number of IHPIs for a particular method forming a DNCC is too high then it impacts negatively the undetectability of the whole DNCC. Considering above, it this paper we assume that the higher the number of IHPIs inflicted by its corresponding covert channels the higher the chance that it will be spotted.

During experiments conducted in this paper we utilize a DNCC which uses multiple steganographic methods that combines various network covert channels. Figure 2 presents such a scenario. The following three subsections present functioning and implementation details of the utilized steganographic methods which we denote in the remaining of this paper as: 'TTL Modulation', 'HTTP Header Reorder' and 'TCP Options Reorder'. To be more specific, this means that for the first method we conceal information by modulating the TTL field value within the IPv4 header, for the second the order of the headers within the HTTP message is influenced, whereas for the third covert channel we embed secret data into the Options field of the TCP header.

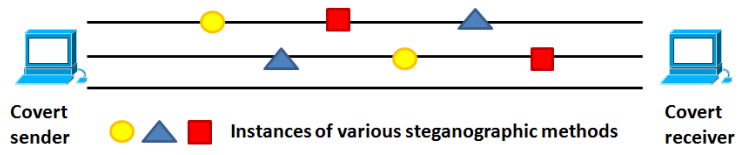

Figure 2: Distributed network covert channel used during conducted experiments.

\subsection{TTL Modulation}

The first utilized steganographic method, which modifies TLL, works as follows. When a new connection suitable for the covert data transfer is established the first few packets are left unmodified. This allows the covert receiver to measure the expected value of the TTL field which depends on the number of intermediate nodes that the packets need to traverse before reaching their destination. This value can be seen as a "hidden data carrier" - subtracting it from the actual TTL in all consecutive packets within the connection reveals the differences where the covert data is embedded. In our data hiding technique a particular value of the TTL difference is mapped to a bit or a group of bits and thus it forms a symbol. We modify the TTL field value within the connection by adding or subtracting a given symbol from its current value. For example, with the expected $\mathrm{TTL}=64$, a change of +5 (i.e. $\mathrm{TTL}=69$ ) denotes a binary 1 whereas a modification of -5 (i.e. TTL $=59)$ denotes binary 0. If the packet carries no secret data then the TTL value is left unchanged. By providing $2^{n}$ symbols our method allows to insert $n$ bits of secret data per packet. For example, a list of symbols: -5 , $-10,+5,+10$ would be mapped to the following TTL values: 59 , $54,69,74$ and the secret data binary values: $00,01,10,11$. Note, that we transmit the secret data by dividing it into chunks of $n$ bits and by mapping their binary values into symbols. This allows to potentially embed up to 8 bits of secret data per packet 
(thus filling the entire TTL field). However, in practice a modification leading to a too small TTL value may cause the packet to be dropped by the routers before reaching its destination. Additionally, significant and frequent changes of the TTL field over a broad range of values may raise suspicion and render the method easily detectable. By observing the characteristics of the benign real-life network traffic, we conclude that no more than 4 bits of secret data could be transmitted in a single packet and the symbols should not exceed a difference of \pm 10 over the expected TTL value.

In the approach that we took in this paper, i.e., by utilizing Software-Defined Networking (SDN) concept for implementing covert communication (for details see subsection 3.1) we can spread the secret data between multiple available TCP connections for the increased stealthiness without significantly degrading covert data transmission rate.

\subsection{HTTP Header Reorder}

The second steganographic method utilizes overt HTTP protocol messages as a hidden data carrier. Covert communication is implemented by tampering with HTTP protocol messages as follows. We capture the HTTP requests and modify them on the fly by modifying the order of the HTTP headers. By changing the sequence of headers in the request and not the data contained within headers, the request itself stays compliant with the HTTP standard. The current HTTP/1.1 standard does not specify any requirements for ordering the headers within requests and responses. In practice this means that the headers are sent in order in which they are stored in the client and server memory - usually a hashmap. During our research we have estimated the number of changes to the order of the headers occurring within a normal HTTP traffic by browsing the Alexa's Top 5000 websites ${ }^{1}$. It turned out, that in the typical user traffic the order of headers changed quite frequently, i.e., in about $18 \%$ of all cases. All common HTTP server software uses request parsers that store the headers in an unordered map, thus, they ignore the discrepancies in the header order and cannot distinguish the difference between a normal HTTP request and a modified one. Our data hiding algorithm assumes that repeating the HTTP requests (sent by the same browser and on the same domain and URL) preserves the same set and the order of the HTTP headers. With such an assumption we establish a baseline by recording the original order of the headers sent on a given URL and compare the subsequent requests with the original one. If there is any difference in the order, we can easily compare the expected and actual order within the packet. The data is encoded by the index of header within the original HTTP request that is relocated into the first (0) position. The index is treated as a symbol which represents a group of bits. The number of headers present in the request determines how many different symbols are possible, which in turn influences how many bits a single symbol can represent. The symbol list can be configured by the user (i.e. the user can work with less symbols than the maximum available). The number of symbols must be a power of 2, i.e., to send 1 bit of covert data you need to specify 2 symbols, for 2 bits you need 4 symbols, for 3 bits -8 symbols, etc. For example, we can send 2 bits of secret data per request by

\footnotetext{
${ }^{1}$ https://www.alexa.com/topsites
}

specifying 4 symbols: 1, 2, 3, 4 which map to $00,01,10,11$ bits. If we consider, for example, the original HTTP request as:

GET /index.html HTTP/1.1

(0) User-Agent: Mozilla/5.0 (Unknown; Linux x86_64)

AppleWebKit/538.1 (KHTML, like Gecko)

PhantomJS/2.1.3-dev-release Safari/538.1

(1) Host: keepalive_on.sdn

(2) Accept-Language: en-us

(3) Accept-Encoding: gzip, deflate

(4) Connection: Keep-Alive

and if we want to encode symbol " 2 ", the header with index 2 (Accept-Language) should get relocated to the beginning, resulting in a request:

GET /index.html HTTP/1.1

(0) Accept-Language: en-us

(1) User-Agent: Mozilla/5.0 (Unknown; Linux x86_64)

AppleWebKit/538.1 (KHTML, like Gecko)

PhantomJS/2.1.3-dev-release Safari/538.1

(2) Host: keepalive_on.sdn

(3) Accept-Encoding: gzip, deflate

(4) Connection: Keep-Alive

The server software treats the modified request as identical to the original one. Additionally, this request still looks legitimate for a human manually inspecting the network traffic. If there is no change of the HTTP headers order it simply means that there is no data encoded within this message.

\subsection{TCP Options Reorder}

For this network steganography technique the covert communication is implemented by tampering with the TCP segments that carry HTTP messages sent to our webserver. We capture the HTTP requests and modify the TCP header of these packets on the fly by changing the order of TCP options.

Because the order of options (apart from option 0, i.e., END OF OPTIONS, which terminates the list and has to be at the end) contained in the non-mandatory TCP options field is not strictly defined, it is possible to send hidden data by rearranging the entries present in the packet without breaking or altering the content it carries in any way.

Due to the existence of many different implementations of the network stack on machines used in the Internet we suspect that option rearrangement occurs with some frequency even in the user benign traffic. If the assumption is correct, this would mean that our data transmission is harder to be spotted and thus more viable. During our research we have assessed the number of changes to the order occurring within normal the HTTP traffic by browsing the Alexa's Top 5000 websites. It turned out that changes to the order of options do, in fact, occur quite frequently (i.e. $2.5 \%$ from 2 billion packets tracked) within user normal traffic, especially with the order of option no. 8 (TIMESTAMP) and no. 1 (NO OPERATION, i.e., a null option used for padding). That is why, we have chosen the order of these two fields as a hidden data carrier for the secret data.

Our algorithm completely ignores packets that do not contain both TCP option no. 8 and TCP option no. 1. If both are present, the algorithm checks if these options follow the initial "default" order 
which was arbitrarily chosen by us to be $(1,8)$. If not, these options are swapped. It should be noted that only the order is checked and not the adjacency. This is because there could be other TCP options present in the list between the two that are used within our data hiding method. It is also possible that the options list contains more than one option no. 1 entry. That is why, the algorithm works only on the first option no. 1 entry found in the list. The order of these two options is preserved until a bit transfer is requested. If this happens the order is swapped to indicate that the bit transfer is about to occur. If the transferred bit carries 0 value, the next packet also has these options swapped (i.e. the same order as in the "reference" packet), which then becomes the "default" order until another bit transfer occurs. If the transferred bit is 1 , the next packet has the TCP options in the same order as the "default" one and the "default" order remains the same.

For example, for the sequence of packets like presented below three secret bits would be transmitted:

(1) order $(1,8)$ - nothing

(2) order $(1,8)$ - nothing

(3) order $(8,1)$ - next packet contains secret data!

(4) order $(8,1)$ - encoded bit: 0

(5) order $(8,1)$ - nothing (the default order has changed)

(6) order $(8,1)$ - nothing

(7) order $(1,8)$ - next packet contains secret data!

(8) order $(8,1)$ - encoded bit: 1

(9) order $(8,1)$ - nothing (the default order has not changed)

(10) order $(1,8)$ - next packet contains secret data!

(11) order $(1,8)$ - encoded bit: 0

(12) order $(1,8)$ - nothing (the default order has changed again)

(13) order $(1,8)$ - nothing

For the network covert channels presented in this section in the remaining of the paper we present our experimental DNCC evaluation and obtained results.

\section{EXPERIMENTAL EVALUATION AND RESULTS}

The main aim of the conducted experiments is to determine the performance and stealthiness of the steganographic methods that are used within the DNCC. Our previous theoretical works [3, 8] proved that utilization of the distributed network covert channels could ensure usable steganographic bandwidth. Moreover, if the diverse steganographic methods are applied within the DNCC then only slight modifications are introduced to the network traffic characteristics that could be easily missed by the security systems. Thus the stealthiness of the whole setup can be increased when compared with the scenario in which only a single data hiding technique is utilized to achieve the same steganographic bandwidth. To obtain reasonable results we decided to perform experimental evaluation by analyzing steganographic methods' performance and stealthiness.

In the remaining of this section we introduce the description of the prepared test-bed and obtained experimental results.

\subsection{Experimental Test-bed}

Due to fact that there is no open source DNCC implementation during our research we developed the dedicated software which is based on the SDN architecture. Such an approach allows separation of the overt traffic and steganographic methods which utilize it to embed secret data. Due to this fact, we prepared the whole SDN setup with separate machines used for sending and receiving the overt traffic as well as machines responsible for embedding and extracting covert data. All utilized machines are virtual ones hosted by the KVM hypervisor.

Three developed steganographic methods are implemented as so called SDN-applications (modules). This code is executed in the SDN controller, which directly interacts with the network devices and passing traffic. Like in the case of the host machines, we utilized virtual network devices using OVS switch. Figure 3 presents the test-bed used during our experiments.

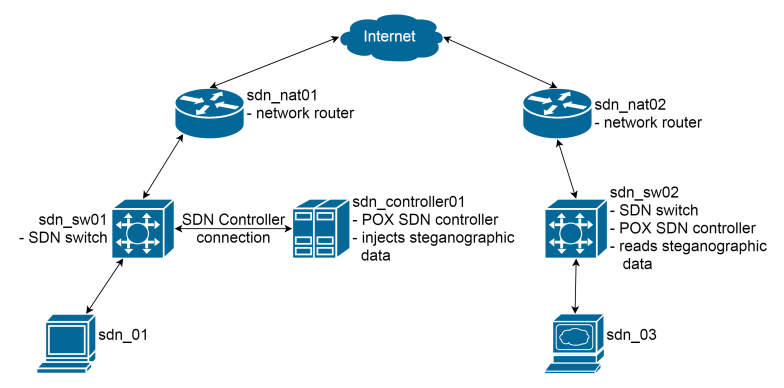

Figure 3: Experimental test-bed.

\subsection{Experimental methodology}

During conducted experiments we embed secret data using custom SDN controller software executed on machine sdn_controller01. As discussed in the previous sections for the DNCC we concurrently use three rather straightforward network covert channels: TTL modulation, HTTP Header Reorder and TCP Options Reorder (see Sect. 2 for details). As an overt traffic we utilize HTTP-based transmissions. During each automated experiment test-run, Chrome headless web browser executed on sdn_01 client machine visits web page hosted on the sdn_03. During our experiments we use a dedicated web server, however, any server in the Internet can be utilized as well. The only requirement is that it should host appropriate number of resources (i.e. web pages or images) for which the number of generated HTTP messages is significant allowing insertion of the configured number of steganographic symbols. Each experiment lasted ca. one minute and depending on the steganographic methods' parameters used from about 5 bytes to 200 bytes of covert data were transmitted.

DNCC detection approach that we utilize in this paper is similar to the approach described in [3]. Like in that article we generate IHPIs from the raw network traffic and then try to identify frequent sets on such data. Each IHPI generated in such a way is described by the item set containing details concerning the source and destination addresses and ports, as well as the type of detected IHPI. Well-known data mining parameter called support (later in the text abbreviated as "sup") defines the number of initial item sets that contain (support) particular set. Parameter called minimal support is used for setting the threshold for detection of frequent set. In other words, we call the set as frequent set when in the initial data 
this set is supported by at least minimal support sets. Due to the fact that DNCC utilizes multiple steganographic methods we decided that our detection system classifies network traffic as containing covert data in the case when at least one frequent set is detected (which describes discovery of the steganographic traffic for a single method). Initial experiments with only one method used, revealed that under normal circumstances in our test environment normal TCP protocol operation generates around 50 events that are flagged as TCP option reorder IHPI. Due to this behavior we decided that the minimal support parameter should be set to at last 50 . Such an assumption allows to reduce the number of false positives.

\subsection{Results}

In this subsection we present the obtained experimental results for two considered strategies: careless where we more or less randomly select the configuration of the DNCC in order to try to maximize its performance without considering how IHPIs are distributed along the covert channels; smart where we try to balance the IHPIs caused by each data hiding technique within DNCC. The motivation for this research comes from our previous experiences related to the detection of DNCC communication where it was revealed that the data mining-based detection can be successful even if the number of IHPIs for only a single data hiding method within the DNCC exceeds a certain threshold. All the details concerning detection of the steganographic traffic using frequent sets can be found in [3].

3.3.1 Careless approach. In the first approach we simply use all available steganographic methods within DNCC with various parameters set. During this part of our experiments we investigated 1,900 variants of parameters sets and here we present only a small fraction of the most interesting results. We sort the obtained results using overall steganographic bandwidth. After this step we are able to choose the DNCC configurations which are most appropriate from the steganographer's perspective. Exemplary results achieved for such an approach for the highest and lowest total DNCC throughput are presented in Tabs. 1 and 2, respectively.

The first three columns in both tables describe details of configuration of the utilized steganographic methods within DNCC:

(1) the names of the steganographic methods used within DNCC,

(2) the number of secret bits per steganographic symbol. For example, '3; 2; 1' means that the TTL-based method is applying 3 secret bits per symbol, HTTP Reorder is embedding 2 bits while the TCP Options-based technique inserts 1 bit,

(3) the frequency of symbols appearance which defines how often packets are steganographically modified. For example, ' $100 ; 5 ; 100$ ' is interpreted as: for the TTL-based method a single steganographic symbol is applied every ca. 100 packets, for HTTP Reorder it is every 5 HTTP messages while for the TCP Options-based it is again a single symbol every 100 packets.

The effective total throughput achieved for such a DNCC using above mentioned parameters is presented in the forth column. Then the next four columns (5-8) illustrate statistics concerning details of the traffic, both overt and covert: the number of used TCP connections, overall overt packet count, the number of steganographically modified packets, and the number of transmitted secret bits. Note that columns 13-15 present different IHPI ratios like IHPIs per bit, per connections or per modified packets. Also in the column 16 result of calculation of the coefficient of variation is placed which is used to conveniently compare various DNCC configuration (see Sec. 3.3.3 for details).

However, the most important results from this paper's perspective are enclosed in the columns 9 - 12 where the number of introduced IHPIs per covert channel used and in total are presented. As mentioned earlier the number of IHPIs is crucial from the perspective of steganographic communication detection as the more IHPIs are present (i.e., the more frequently the hidden data carrier is modified) the more opportunities for spotting DNCC utilization. As visible in Tab. 1, DNCC with parameters like in the first row (i.e., 3; 2; 1 bit/symbol and 100; 5; 100 symbol frequency) achieves the highest overall throughput but uses ca. 1000 IHPIs related to the TCP Options Reorder method. This is a noticeably higher number than the number of IHPIs used by the other two techniques. Thus, such a situation can lead to easier detection of the DNCC due to excessive usage of a single steganographic technique. However, when we look at the last two rows in Tab. 1 we can observe that only a bit smaller steganographic bandwidth can be achieved with lower number of IHPIs related to the TCP Options Reorder and HTTP Header Reorder covert channels. Moreover, in such parameters sets IHPIs associated to both steganographic methods are the lowest ones.

Similar situation is visible when we consider rather slow but more stealthy communication of which results are presented in the Tab. 2. In the first three rows we can discover that for such DNCC configuration more than 120 IHPIs related to the HTTP header method are utilized, in contrast to no more than 60 IHPIs for remaining two techniques. Like already mentioned for the previously presented results in the Tab. 2 we can also point out parameters for which the number of IHPIs for all methods are more balanced. For example, we are able to establish 8 parameter sets with less than 100 IHPI for the HTTP method.

To summarize, it must be noted that in most cases setting parameters in a careless manner leads to the situation where IHPIs number of one method belonging to the DNCC is noticeable larger than the other two. Such imbalanced situation can compromise DNCC stealthiness. In effect, covert communication can be spotted easily only by detecting a single steganographic method which is used excessively and introduces noticeably larger number of IHPIs. That is why, in the following subsection we investigate a different strategy which can help ensure increased stealthiness of the DNCC created using covert channels characterized with various steganographic bandwidth.

3.3.2 Detection of the careless approach. The second part of our research is based on the observations made in the previous subsection as well as based on the experiences from our past work. As already mentioned, in [3] we established that using data miningbased steganalysis can be successful even if the number of IHPIs for only a single data hiding techniques within DNCC exceeds a certain threshold. This means that if utilized naively then DNCC can be spotted quite easily. In the remaining of this subsection we will show that if the DNCC is configured in a careless manner then its detection is rather straightforward. 
Table 1: The obtained results for the DNCC utilizing three separate steganographic methods - highest throughput (careless approach)

\begin{tabular}{|c|c|c|c|c|c|c|c|c|c|c|c|c|c|c|c|}
\hline \multicolumn{4}{|c|}{ DNCC parameters } & \multicolumn{4}{|c|}{ Traffic statistics (at Covert Sender) } & \multicolumn{4}{|c|}{ Number of IHPIs } & \multicolumn{3}{|c|}{ Ratios } & \multirow[b]{2}{*}{$\mathrm{CV}$} \\
\hline methods used & $\begin{array}{c}\text { bit } \\
\text { per } \\
\text { symbol }\end{array}$ & $\begin{array}{l}\text { symbol } \\
\text { freq. }\end{array}$ & $\begin{array}{l}\text { steg. } \\
\text { band. } \\
\text { [bps] }\end{array}$ & $\begin{array}{c}\mathrm{TCP} \\
\text { connections }\end{array}$ & $\begin{array}{l}\text { overall } \\
\text { packet } \\
\text { count }\end{array}$ & $\begin{array}{c}\text { modified } \\
\text { packet } \\
\text { count }\end{array}$ & $\begin{array}{c}\text { secret } \\
\text { bits } \\
\text { transmitted }\end{array}$ & $\begin{array}{c}\text { TTL } \\
\text { change }\end{array}$ & $\begin{array}{l}\text { TCP opt } \\
\text { reorder }\end{array}$ & $\begin{array}{l}\text { HTTP } \\
\text { header } \\
\text { reorder }\end{array}$ & Sum & $\begin{array}{c}\text { IHPIs per } \\
\text { bit }\end{array}$ & $\begin{array}{l}\text { IHPIs per } \\
\text { connection }\end{array}$ & $\begin{array}{l}\text { IHPIs per } \\
\text { p.modified }\end{array}$ & \\
\hline TTL; HTTP Reorder; TCP opt. & $3,2,1$ & $100,5,100$ & 27.475 & 102.0 & 59443.5 & 823.50 & 1648.5 & 691.5 & 998.5 & 400.0 & 2090.00 & 1.265 & 20.49 & 2.54 & 0.351 \\
\hline TTL; HTTP Reorder; TCP opt. & $3,2,1$ & $100,10,100$ & 25.475 & 102.0 & 59419.0 & 763.50 & 1528.5 & 693.5 & 996.0 & 501.5 & 2191.00 & 1.435 & 21.48 & 2.87 & 0.278 \\
\hline TTL; HTTP Reorder; TCP opt. & $3,1,1$ & $100,5,100$ & 25.365 & 102.0 & 59541.0 & 827.00 & 1522.0 & 695.5 & 926.5 & 235.5 & 1857.50 & 1.220 & 18.21 & 2.25 & 0.464 \\
\hline TTL; HTTP Reorder; TCP opt. & $\begin{array}{l}3,1,1,1 \\
3,2\end{array}$ & $100,15,100$ & 24.715 & 102.0 & 59248.5 & 741.00 & 1483.0 & 692.0 & 999.0 & 343.0 & 2034.00 & 1.375 & 19.94 & 2.75 & 0.395 \\
\hline TTL; HTTP Reorder; TCP opt. & $3,1,1$ & $100,10,100$ & 24.340 & 102.0 & 59483.0 & 765.50 & 1460.5 & 696.0 & 993.0 & 351.5 & 2040.50 & 1.395 & 20.01 & 2.67 & 0.385 \\
\hline TTL; HTTP Reorder; TCP opt. & $\begin{array}{l}3,1,1 \\
3,2,1\end{array}$ & $100,20,100$ & 24.170 & 102.0 & 59052.0 & 724.00 & 1450.0 & 690.0 & 980.0 & 261.0 & 1931.00 & 1.330 & 18.93 & 2.67 & 0.459 \\
\hline TTL; HTTP Reorder; TCP opt. & $3,2,1$ & $100,25,100$ & 24.100 & 102.0 & 59212.0 & 722.00 & 1446.0 & 694.0 & 938.0 & 228.0 & 1860.00 & 1.290 & 18.24 & 2.58 & 0.475 \\
\hline TTL; HTTP Reorder; TCP opt. & $3,2,1$ & $100,30,100$ & 23.950 & 102.0 & 59464.0 & 718.00 & 1437.0 & 696.0 & 980.0 & 153.0 & 1829.00 & 1.270 & 17.93 & 2.55 & 0.563 \\
\hline TTL; HTTP Reorder; TCP opt. & $3,1,1$ & $100,15,100$ & 23.915 & 101.0 & 59300.0 & 742.00 & 1435.0 & 695.0 & 918.0 & 240.0 & 1853.00 & 1.290 & 18.34 & 2.50 & 0.457 \\
\hline TTL; HTTP Reorder; TCP opt. & $3,2,1$ & $100,40,100$ & 23.820 & 102.0 & 59622.0 & 714.00 & 1429.0 & 696.0 & 996.0 & 122.0 & 1814.00 & 1.270 & 17.78 & 2.54 & 0.600 \\
\hline TTL; HTTP Reorder; TCP opt. & $\begin{array}{l}3,2,1 \\
3,2,1\end{array}$ & $100,35,100$ & 23.780 & 102.0 & 59278.0 & 713.00 & 1427.0 & 692.0 & 999.0 & 152.0 & 1843.00 & 1.290 & 18.07 & 2.58 & 0.570 \\
\hline TTL; HTTP Reorder; TCP opt. & $3,1,1$ & $100,20,100$ & 23.680 & 102.0 & 59083.0 & 729.00 & 1421.0 & 692.0 & 973.0 & 180.0 & 1845.00 & 1.300 & 18.09 & 2.53 & 0.534 \\
\hline TTL; HTTP Reorder; TCP opt. & $3,1,1$ & $100,25,100$ & 23.670 & 100.0 & 59347.0 & 724.00 & 1420.0 & 698.0 & 1046.0 & 156.0 & 1900.00 & 1.340 & 19.00 & 2.62 & 0.578 \\
\hline TTL; HTTP Reorder; TCP opt. & $3,2,1$ & $100,45,100$ & 23.600 & 102.0 & 59490.0 & 707.00 & 1416.0 & 692.0 & 936.0 & 114.0 & 1742.00 & 1.230 & 17.08 & 2.46 & 0.594 \\
\hline TTL; HTTP Reorder; TCP opt. & $3,1,1$ & $100,45,100$ & 23.400 & 102.0 & 59610.0 & 710.00 & 1404.0 & 696.0 & 919.0 & 82.0 & 1697.00 & 1.210 & 16.64 & 2.39 & 0.626 \\
\hline
\end{tabular}

Table 2: The obtained results for the DNCC utilizing three separate steganographic methods - lowest throughput (careless approach)

\begin{tabular}{|c|c|c|c|c|c|c|c|c|c|c|c|c|c|c|c|}
\hline \multicolumn{4}{|c|}{ DNCC parameters } & \multicolumn{4}{|c|}{ Traffic statistics (at Covert Sender) } & \multicolumn{4}{|c|}{ Number of IHPIs } & \multicolumn{3}{|c|}{ Ratios } & \multirow[b]{2}{*}{$\mathrm{CV}$} \\
\hline methods used & $\begin{array}{c}\text { bit } \\
\text { per } \\
\text { symbol }\end{array}$ & $\begin{array}{l}\text { symbol } \\
\text { freq. }\end{array}$ & $\begin{array}{l}\text { steg. } \\
\text { band. } \\
\text { [bps] }\end{array}$ & $\begin{array}{c}\text { TCP } \\
\text { connections }\end{array}$ & $\begin{array}{l}\text { overall } \\
\text { packet } \\
\text { count }\end{array}$ & $\begin{array}{l}\text { modified } \\
\text { packet } \\
\text { count }\end{array}$ & $\begin{array}{c}\text { secret } \\
\text { bits } \\
\text { transmitted }\end{array}$ & $\begin{array}{c}\text { TTL } \\
\text { change }\end{array}$ & $\begin{array}{l}\text { TCP opt } \\
\text { reorder }\end{array}$ & $\begin{array}{l}\text { HTTP } \\
\text { header } \\
\text { reorder }\end{array}$ & Sum & $\begin{array}{c}\text { IHPIs per } \\
\text { bit }\end{array}$ & $\begin{array}{l}\text { IHPIs per } \\
\text { connection }\end{array}$ & $\begin{array}{l}\text { IHPIs per } \\
\text { p.modified }\end{array}$ & \\
\hline TTL; HTTP Reorder; TCP opt. & $1,1,1$ & $2100,30,1600$ & 1.050 & 102.0 & 59531.0 & 63.00 & 63.0 & 32.0 & 60.0 & 125.0 & 217.00 & 3.440 & 2.13 & 3.44 & 0.539 \\
\hline TTL; HTTP Reorder; TCP opt. & $1,1,1$ & $2100,25,2100$ & 1.030 & 102.0 & 59704.0 & 62.00 & 62.0 & 32.0 & 44.0 & 162.0 & 238.00 & 3.840 & 2.33 & 3.84 & 0.739 \\
\hline TTL; HTTP Reorder; TCP opt. & $1,1,1$ & $1600,30,2100$ & 1.030 & 102.0 & 59478.0 & 62.00 & 62.0 & 44.0 & 50.0 & 122.0 & 216.00 & 3.480 & 2.12 & 3.48 & 0.492 \\
\hline TTL; HTTP Reorder; TCP opt. & $1,1,1$ & $1600,40,1600$ & 1.030 & 102.0 & 59490.0 & 62.00 & 62.0 & 44.0 & 64.0 & 92.0 & 200.00 & 3.230 & 1.96 & 3.23 & 0.295 \\
\hline TTL; HTTP Reorder; TCP opt. & $1,1,1$ & $1600,45,1600$ & 1.020 & 102.0 & 59648.0 & 61.00 & 61.0 & 44.0 & 66.0 & 77.0 & 187.00 & 3.070 & 1.83 & 3.07 & 0.220 \\
\hline TTL; HTTP Reorder; TCP opt. & $1,1,1$ & $2100,35,1600$ & 0.980 & 102.0 & 59378.0 & 59.00 & 59.0 & 32.0 & 66.0 & 101.0 & 199.00 & 3.370 & 1.95 & 3.37 & 0.425 \\
\hline TTL; HTTP Reorder; TCP opt. & $1,1,1$ & $1600,35,2100$ & 0.980 & 102.0 & 59075.0 & 59.00 & 59.0 & 42.0 & 48.0 & 104.0 & 194.00 & 3.290 & 1.90 & 3.29 & 0.432 \\
\hline TTL; HTTP Reorder; TCP opt. & $1,1,1$ & $2100,30,2100$ & 0.950 & 102.0 & 59469.0 & 57.00 & 57.0 & 32.0 & 50.0 & 114.0 & 196.00 & 3.440 & 1.92 & 3.44 & 0.539 \\
\hline TTL; HTTP Reorder; TCP opt. & $1,1,1$ & $2100,40,1600$ & 0.950 & 102.0 & 59516.0 & 57.00 & 57.0 & 32.0 & 58.0 & 87.0 & 177.00 & 3.110 & 1.74 & 3.11 & 0.381 \\
\hline TTL; HTTP Reorder; TCP opt. & $1,1,1$ & $1600,40,2100$ & 0.930 & 102.0 & 59620.0 & 56.00 & 56.0 & 42.0 & 50.0 & 87.0 & 179.00 & 3.200 & 1.75 & 3.20 & 0.329 \\
\hline TTL; HTTP Reorder; TCP opt. & $1,1,1$ & $2100,45,1600$ & 0.900 & 102.0 & 58810.0 & 54.00 & 54.0 & 32.0 & 65.0 & 78.0 & 175.00 & 3.240 & 1.72 & 3.24 & 0.332 \\
\hline TTL; HTTP Reorder; TCP opt. & $1,1,1$ & $1600,45,2100$ & 0.900 & 102.0 & 59048.0 & 54.00 & 54.0 & 42.0 & 48.0 & 74.0 & 164.00 & 3.040 & 1.61 & 3.04 & 0.254 \\
\hline TTL; HTTP Reorder; TCP opt. & $1,1,1$ & $2100,35,2100$ & 0.900 & 102.0 & 59688.0 & 54.00 & 54.0 & 32.0 & 48.0 & 100.0 & 180.00 & 3.330 & 1.76 & 3.33 & 0.484 \\
\hline TTL; HTTP Reorder; TCP opt. & $1,1,1$ & $2100,40,2100$ & 0.850 & 100.0 & 59545.0 & 51.00 & 51.0 & 32.0 & 46.0 & 86.0 & 164.00 & 3.220 & 1.64 & 3.22 & 0.419 \\
\hline TTL; HTTP Reorder; TCP opt. & $1,1,1$ & $2100,45,2100$ & 0.820 & 100.0 & 59111.0 & 49.00 & 49.0 & 32.0 & 44.0 & 79.0 & 155.00 & 3.160 & 1.55 & 3.16 & 0.386 \\
\hline
\end{tabular}

Table 3: Detection of the careless approach-based DNCC using frequent sets approach (for minsup=50) - highest throughput.

\begin{tabular}{|c|c|c|c|c|c|c|c|c|c|c|c|c|c|c|c|}
\hline \multicolumn{4}{|c|}{ DNCC parameters } & \multicolumn{4}{|c|}{ Traffic statistics (at Covert Sender) } & \multicolumn{4}{|c|}{ Summary support } & \multicolumn{3}{|c|}{ Ratios } & \multirow[b]{2}{*}{$\mathrm{CV}$} \\
\hline methods used & $\begin{array}{c}\text { bit } \\
\text { per } \\
\text { symbol }\end{array}$ & $\begin{array}{l}\text { symbol } \\
\text { freq. }\end{array}$ & $\begin{array}{l}\text { steg. } \\
\text { band. } \\
\text { [bps] }\end{array}$ & $\begin{array}{c}\text { TCP } \\
\text { connections }\end{array}$ & $\begin{array}{c}\text { overall } \\
\text { packet } \\
\text { count }\end{array}$ & $\begin{array}{c}\text { modified } \\
\text { packet } \\
\text { count }\end{array}$ & $\begin{array}{c}\text { secret } \\
\text { bits } \\
\text { transmitted }\end{array}$ & $\begin{array}{c}\text { TTL } \\
\text { change }\end{array}$ & $\begin{array}{l}\text { TCP opt } \\
\text { reorder }\end{array}$ & $\begin{array}{l}\text { HTTP } \\
\text { header } \\
\text { reorder }\end{array}$ & Sum & $\begin{array}{c}\text { IHPIs per } \\
\text { bit }\end{array}$ & $\begin{array}{l}\text { IHPIs per } \\
\text { connection }\end{array}$ & $\begin{array}{l}\text { IHPIs per } \\
\text { p.modified }\end{array}$ & \\
\hline TTL; HTTP Reorder; TCP opt. & $3,2,1$ & $100,5,100$ & 27.475 & 102.0 & 59443.5 & 823.50 & 1648.5 & 613.0 & 402.5 & 138.5 & 1154.00 & 0.700 & 11.31 & 1.40 & 0.505 \\
\hline TTL; HTTP Reorder; TCP opt. & $3,2,1$ & $100,10,100$ & 25.475 & 102.0 & 59419.0 & 763.50 & 1528.5 & 616.5 & 399.5 & 138.5 & 1154.50 & 0.755 & 11.32 & 1.52 & 0.510 \\
\hline TTL; HTTP Reorder; TCP opt. & $3,1,1$ & $100,5,100$ & 25.365 & 102.0 & 59541.0 & 827.00 & 1522.0 & 621.0 & 367.5 & 110.0 & 1098.50 & 0.720 & 10.77 & 1.33 & 0.570 \\
\hline TTL; HTTP Reorder; TCP opt. & $3,2,1$ & $100,15,100$ & 24.715 & 102.0 & 59248.5 & 741.00 & 1483.0 & 621.0 & 407.5 & 97.5 & 1126.00 & 0.760 & 11.04 & 1.52 & 0.573 \\
\hline TTL; HTTP Reorder; TCP opt. & $3,1,1$ & $100,10,100$ & 24.340 & 102.0 & 59483.0 & 765.50 & 1460.5 & 618.0 & 404.5 & 139.0 & 1161.50 & 0.795 & 11.38 & 1.52 & 0.506 \\
\hline TTL; HTTP Reorder; TCP opt. & $3,2,1$ & $100,20,100$ & 24.170 & 102.0 & 59052.0 & 724.00 & 1450.0 & 622.0 & 394.0 & 75.0 & 1091.00 & 0.750 & 10.70 & 1.51 & 0.617 \\
\hline TTL; HTTP Reorder; TCP opt. & $3,2,1$ & $100,25,100$ & 24.100 & 102.0 & 59212.0 & 722.00 & 1446.0 & 618.0 & 374.0 & 65.0 & 1057.00 & 0.730 & 10.36 & 1.46 & 0.642 \\
\hline TTL; HTTP Reorder; TCP opt. & $3,2,1$ & $100,30,100$ & 23.950 & 102.0 & 59464.0 & 718.00 & 1437.0 & 620.0 & 434.0 & 0.0 & 1054.00 & 0.730 & 10.33 & 1.47 & 0.739 \\
\hline TTL; HTTP Reorder; TCP opt. & $3,1,1$ & $100,15,100$ & 23.915 & 101.0 & 59300.0 & 742.00 & 1435.0 & 613.0 & 346.5 & 96.5 & 1056.00 & 0.735 & 10.46 & 1.42 & 0.599 \\
\hline TTL; HTTP Reorder; TCP opt. & $3,2,1$ & $100,40,100$ & 23.820 & 102.0 & 59622.0 & 714.00 & 1429.0 & 626.0 & 441.0 & 0.0 & 1067.00 & 0.750 & 10.46 & 1.49 & 0.738 \\
\hline TTL; HTTP Reorder; TCP opt. & $3,2,1$ & $100,35,100$ & 23.780 & 102.0 & 59278.0 & 713.00 & 1427.0 & 608.0 & 407.0 & 0.0 & 1015.00 & 0.710 & 9.95 & 1.42 & 0.748 \\
\hline TTL; HTTP Reorder; TCP opt. & $3,1,1$ & $100,20,100$ & 23.680 & 102.0 & 59083.0 & 729.00 & 1421.0 & 618.0 & 383.0 & 74.0 & 1075.00 & 0.760 & 10.54 & 1.47 & 0.622 \\
\hline TTL; HTTP Reorder; TCP opt. & $3,1,1$ & $100,25,100$ & 23.670 & 100.0 & 59347.0 & 724.00 & 1420.0 & 628.0 & 428.0 & 65.0 & 1121.00 & 0.790 & 11.21 & 1.55 & 0.624 \\
\hline TTL; HTTP Reorder; TCP opt. & $3,2,1$ & $100,45,100$ & 23.600 & 102.0 & 59490.0 & 707.00 & 1416.0 & 630.0 & 375.0 & 0.0 & 1005.00 & 0.710 & 9.85 & 1.42 & 0.772 \\
\hline TTL; HTTP Reorder; TCP opt. & $3,1,1$ & $100,45,100$ & 23.400 & 102.0 & 59610.0 & 710.00 & 1404.0 & 624.0 & 369.0 & 0.0 & 993.00 & 0.710 & 9.74 & 1.40 & 0.774 \\
\hline
\end{tabular}

Note, that in this paper we utilize a similar approach for the detection of DNCC as indicated above. During conducted experiments, and more specifically during the pre-processing phase, we generate IHPIs for all three covert channel techniques. In the next step we discover frequent sets. Discovery of the frequent set which contains item set associated with a given steganographic method is a proof of steganographic transmission existence.
Exemplary obtained results for the summary support (for the same configurations as showed for the careless approach) are presented in Tabs. 3 and 4 and discussed below. In Tab. 3 the number of the detected frequent sets for various DNCC configurations are presented. As it can be seen for all of them we are able to detect frequent sets thus the DNCC is detectable (which is depicted with the non-zero values in the summary support part of the table). For example, for the DNCC configuration presented in the first row 
Table 4: Detection of the careless approach-based DNCC using frequent sets approach (for minsup=50) - lowest throughput.

\begin{tabular}{|c|c|c|c|c|c|c|c|c|c|c|c|c|c|c|c|}
\hline \multicolumn{4}{|c|}{ DNCC parameters } & \multicolumn{4}{|c|}{ Traffic statistics (at Covert Sender) } & \multicolumn{4}{|c|}{ Summary support } & \multicolumn{3}{|c|}{ Ratios } & \multirow[b]{2}{*}{$\mathrm{CV}$} \\
\hline methods used & $\begin{array}{c}\text { bit } \\
\text { per } \\
\text { symbol }\end{array}$ & $\begin{array}{l}\text { symbol } \\
\text { freq. }\end{array}$ & $\begin{array}{l}\text { steg. } \\
\text { band. } \\
\text { [bps] }\end{array}$ & $\begin{array}{c}\text { TCP } \\
\text { connections }\end{array}$ & $\begin{array}{l}\text { overall } \\
\text { packet } \\
\text { count }\end{array}$ & $\begin{array}{c}\text { modified } \\
\text { packet } \\
\text { count }\end{array}$ & $\begin{array}{c}\text { secret } \\
\text { bits } \\
\text { transmitted }\end{array}$ & $\begin{array}{c}\text { TTL } \\
\text { change }\end{array}$ & $\begin{array}{l}\text { TCP opt } \\
\text { reorder }\end{array}$ & $\begin{array}{l}\text { HTTP } \\
\text { header } \\
\text { reorder }\end{array}$ & Sum & $\begin{array}{c}\text { IHPIs per } \\
\text { bit }\end{array}$ & $\begin{array}{l}\text { IHPIs per } \\
\text { connection }\end{array}$ & $\begin{array}{l}\text { IHPIs per } \\
\text { p.modified }\end{array}$ & \\
\hline TTL; HTTP Reorder; TCP opt. & $1,1,1$ & $2100,30,1600$ & 1.050 & 102.0 & 59531.0 & 63.00 & 63.0 & 0.0 & 0.0 & 50.0 & 50.00 & 0.790 & 0.49 & 0.79 & 1.414 \\
\hline TTL; HTTP Reorder; TCP opt. & $1,1,1$ & $2100,25,2100$ & 1.030 & 102.0 & 59704.0 & 62.00 & 62.0 & 0.0 & 0.0 & 65.0 & 65.00 & 1.050 & 0.64 & 1.05 & 1.414 \\
\hline TTL; HTTP Reorder; TCP opt. & $1,1,1$ & $1600,30,2100$ & 1.030 & 102.0 & 59478.0 & 62.00 & 62.0 & 0.0 & 0.0 & 0.0 & 0.00 & 0.000 & 0.00 & 0.00 & - \\
\hline TTL; HTTP Reorder; TCP opt. & $1,1,1$ & $1600,40,1600$ & 1.030 & 102.0 & 59490.0 & 62.00 & 62.0 & 0.0 & 0.0 & 0.0 & 0.00 & 0.000 & 0.00 & 0.00 & - \\
\hline TTL; HTTP Reorder; TCP opt. & $1,1,1$ & $1600,45,1600$ & 1.020 & 102.0 & 59648.0 & 61.00 & 61.0 & 0.0 & 0.0 & 0.0 & 0.00 & 0.000 & 0.00 & 0.00 & - \\
\hline TTL; HTTP Reorder; TCP opt. & $1,1,1$ & $2100,35,1600$ & 0.980 & 102.0 & 59378.0 & 59.00 & 59.0 & 0.0 & 0.0 & 0.0 & 0.00 & 0.000 & 0.00 & 0.00 & - \\
\hline TTL; HTTP Reorder; TCP opt. & $1,1,1$ & $1600,35,2100$ & 0.980 & 102.0 & 59075.0 & 59.00 & 59.0 & 0.0 & 0.0 & 0.0 & 0.00 & 0.000 & 0.00 & 0.00 & - \\
\hline TTL; HTTP Reorder; TCP opt. & $1,1,1$ & $2100,30,2100$ & 0.950 & 102.0 & 59469.0 & 57.00 & 57.0 & 0.0 & 0.0 & 0.0 & 0.00 & 0.000 & 0.00 & 0.00 & - \\
\hline TTL; HTTP Reorder; TCP opt. & $1,1,1$ & $2100,40,1600$ & 0.950 & 102.0 & 59516.0 & 57.00 & 57.0 & 0.0 & 0.0 & 0.0 & 0.00 & 0.000 & 0.00 & 0.00 & - \\
\hline TTL; HTTP Reorder; TCP opt. & $1,1,1$ & $1600,40,2100$ & 0.930 & 102.0 & 59620.0 & 56.00 & 56.0 & 0.0 & 0.0 & 0.0 & 0.00 & 0.000 & 0.00 & 0.00 & - \\
\hline TTL; HTTP Reorder; TCP opt. & $1,1,1$ & $2100,45,1600$ & 0.900 & 102.0 & 58810.0 & 54.00 & 54.0 & 0.0 & 0.0 & 0.0 & 0.00 & 0.000 & 0.00 & 0.00 & - \\
\hline TTL; HTTP Reorder; TCP opt. & $1,1,1$ & $1600,45,2100$ & 0.900 & 102.0 & 59048.0 & 54.00 & 54.0 & 0.0 & 0.0 & 0.0 & 0.00 & 0.000 & 0.00 & 0.00 & - \\
\hline TTL; HTTP Reorder; TCP opt. & $1,1,1$ & $2100,35,2100$ & 0.900 & 102.0 & 59688.0 & 54.00 & 54.0 & 0.0 & 0.0 & 0.0 & 0.00 & 0.000 & 0.00 & 0.00 & - \\
\hline TTL; HTTP Reorder; TCP opt. & $1,1,1$ & $2100,40,2100$ & 0.850 & 100.0 & 59545.0 & 51.00 & 51.0 & 0.0 & 0.0 & 0.0 & 0.00 & 0.000 & 0.00 & 0.00 & - \\
\hline TTL; HTTP Reorder; TCP opt. & $1,1,1$ & $2100,45,2100$ & 0.820 & 100.0 & 59111.0 & 49.00 & 49.0 & 0.0 & 0.0 & 0.0 & 0.00 & 0.000 & 0.00 & 0.00 & - \\
\hline
\end{tabular}

Table 5: The obtained results for the DNCC utilizing three separate steganographic methods (smart approach).

\begin{tabular}{|c|c|c|c|c|c|c|c|c|c|c|c|c|c|c|c|}
\hline \multicolumn{4}{|c|}{ DNCC parameters } & \multicolumn{4}{|c|}{ Traffic statistics (at Covert Sender) } & \multicolumn{4}{|c|}{ Number of IHPIs } & \multicolumn{3}{|c|}{ Ratios } & \multirow[b]{2}{*}{$\mathrm{CV}$} \\
\hline methods used & $\begin{array}{c}\text { bit } \\
\text { per } \\
\text { symbol }\end{array}$ & $\begin{array}{l}\text { symbol } \\
\text { freq. }\end{array}$ & $\begin{array}{l}\text { steg. } \\
\text { band. } \\
\text { [bps] }\end{array}$ & $\begin{array}{c}\text { TCP } \\
\text { connections }\end{array}$ & $\begin{array}{l}\text { overall } \\
\text { packet } \\
\text { count }\end{array}$ & $\begin{array}{c}\text { modified } \\
\text { packet } \\
\text { count }\end{array}$ & $\begin{array}{c}\text { secret } \\
\text { bits } \\
\text { transmitted }\end{array}$ & $\begin{array}{c}\text { TTL } \\
\text { change }\end{array}$ & $\begin{array}{l}\mathrm{TCP} \text { opt } \\
\text { reorder }\end{array}$ & $\begin{array}{l}\text { HTTP } \\
\text { header } \\
\text { reorder }\end{array}$ & Sum & $\begin{array}{c}\text { IHPIs per } \\
\text { bit }\end{array}$ & $\begin{array}{l}\text { IHPIs per } \\
\text { connection }\end{array}$ & $\begin{array}{l}\text { IHPIs per } \\
\text { p.modified }\end{array}$ & \\
\hline TTL; HTTP Reorder; TCP opt. & $3,2,1$ & $600,40,600$ & 4.500 & 102.0 & 59496.0 & 135.00 & 270.0 & 115.0 & 148.0 & 145.0 & 408.00 & 1.510 & 4.00 & 3.02 & 0.110 \\
\hline TTL; HTTP Reorder; TCP opt. & $3,2,1$ & $600,45,600$ & 4.430 & 102.0 & 59731.0 & 133.00 & 266.0 & 116.0 & 156.0 & 123.0 & 395.00 & 1.480 & 3.87 & 2.97 & 0.132 \\
\hline TTL; HTTP Reorder; TCP opt. & $3,2,1$ & $600,45,1100$ & 3.980 & 102.0 & 59125.0 & 106.00 & 239.0 & 116.0 & 95.0 & 125.0 & 336.00 & 1.410 & 3.29 & 3.17 & 0.112 \\
\hline TTL; HTTP Reorder; TCP opt. & $3,1,1$ & $600,35,1100$ & 3.770 & 102.0 & 59183.0 & 110.00 & 226.0 & 116.0 & 92.0 & 112.0 & 320.00 & 1.420 & 3.14 & 2.91 & 0.098 \\
\hline TTL; HTTP Reorder; TCP opt. & $3,1,1$ & $600,40,1100$ & 3.750 & 102.0 & 59548.0 & 109.00 & 225.0 & 116.0 & 90.0 & 90.0 & 296.00 & 1.320 & 2.90 & 2.72 & 0.124 \\
\hline TTL; HTTP Reorder; TCP opt. & $2,2,1$ & $600,40,1100$ & 3.100 & 102.0 & 59441.0 & 109.00 & 186.0 & 116.0 & 95.0 & 118.0 & 329.00 & 1.770 & 3.23 & 3.02 & 0.095 \\
\hline TTL; HTTP Reorder; TCP opt. & $2,2,1$ & $600,45,1100$ & 3.030 & 102.0 & 59843.0 & 107.00 & 182.0 & 116.0 & 100.0 & 95.0 & 311.00 & 1.710 & 3.05 & 2.91 & 0.086 \\
\hline TTL; HTTP Reorder; TCP opt. & $2,1,1$ & $600,35,1100$ & 2.830 & 102.0 & 59714.0 & 112.00 & 170.0 & 116.0 & 98.0 & 102.0 & 316.00 & 1.860 & 3.10 & 2.82 & 0.073 \\
\hline TTL; HTTP Reorder; TCP opt. & $2,1,1$ & $600,40,1100$ & 2.780 & 102.0 & 59669.0 & 109.00 & 167.0 & 116.0 & 96.0 & 88.0 & 300.00 & 1.800 & 2.94 & 2.75 & 0.118 \\
\hline TTL; HTTP Reorder; TCP opt. & $1,2,1$ & $600,40,600$ & 2.570 & 102.0 & 59489.0 & 135.00 & 154.0 & 116.0 & 154.0 & 128.0 & 398.00 & 2.580 & 3.90 & 2.95 & 0.120 \\
\hline TTL; HTTP Reorder; TCP opt. & $3,1,1$ & $1100,45,1100$ & 2.420 & 102.0 & 59749.0 & 81.00 & 145.0 & 64.0 & 82.0 & 76.0 & 222.00 & 1.530 & 2.18 & 2.74 & 0.101 \\
\hline TTL; HTTP Reorder; TCP opt. & $1,2,1$ & $600,40,1100$ & 2.120 & 102.0 & 59363.0 & 108.00 & 127.0 & 116.0 & 96.0 & 133.0 & 345.00 & 2.720 & 3.38 & 3.19 & 0.131 \\
\hline TTL; HTTP Reorder; TCP opt. & $1,1,1$ & $600,35,1100$ & 1.870 & 102.0 & 59743.0 & 112.00 & 112.0 & 116.0 & 99.0 & 104.0 & 319.00 & 2.850 & 3.13 & 2.85 & 0.067 \\
\hline TTL; HTTP Reorder; TCP opt. & $2,1,1$ & $1100,45,1600$ & 1.670 & 102.0 & 59317.0 & 69.00 & 100.0 & 62.0 & 62.0 & 80.0 & 204.00 & 2.040 & 2.00 & 2.96 & 0.125 \\
\hline TTL; HTTP Reorder; TCP opt. & $1,1,1$ & $1100,45,1100$ & 1.350 & 102.0 & 59688.0 & 81.00 & 81.0 & 64.0 & 82.0 & 78.0 & 224.00 & 2.770 & 2.20 & 2.77 & 0.103 \\
\hline
\end{tabular}

(i.e., 3; $2 ; 1 \mathrm{bit} / \mathrm{symbol}$ and 100; 5; 100 symbol frequency) we were able to detect almost half of the IHPIs (1154 out of 2090).

However, more interesting results are presented in the Tab. 4. Note, that in this case only in the first two rows some frequent sets have been discovered. Moreover, they are encountered only for one method - HTTP header reorder. In the remaining rows there are no frequent sets discovered, i.e., the introduced DNCC is not detectable by the utilized data mining-based method, however, the resulting steganographic bandwidth for these cases is low, i.e., around $1 \mathrm{bps}$. Therefore, the question arises is it possible to construct the DNCC in such a way that it still remains undetectable but offers increased hidden data rates. We will explore this issue in the next subsection.

3.3.3 Smart approach. Considering experimental results presented in the previous subsection it is visible that in order to develop a more stealthy DNCC one should tune parameters of each of its steganographic method separately in such a way that the number of introduced IHPIs is balanced. In such case, there would not be any noticeable number of IHPIs for a single steganographic method which would be easily detectable by the data mining approach and thus can compromise the overall DNCC stealthiness. Instead, we reviewed all possible configurations of the DNCC test-runs and choose these, which have balanced IHPI numbers.

In order to determine which DNCC configurations result in a balanced approach for each of them we calculate coefficient of variation $(\mathrm{CV})$ which is a well-known statistical measure typically used to express the extent of variability in relation to the mean (relative standard deviation). The higher the coefficient of variation, the greater the level of dispersion around the mean. In our case the lower the $\mathrm{CV}$ for the certain configuration the more balanced approach is. Coefficient of variation is defined as a ratio between the standard deviation of the number of IHPIs for the analyzed steganographic methods and a corresponding mean value. So for example, for the three utilized covert techniques the number of IHPIs is 115,148 , and 145 then the standard deviation is equal to 14.9 , the mean is 136 and thus the resulting $\mathrm{CV}$ is equivalent to 0.11 . Rationale for using CV is that it allows, independently of the order of magnitude of the resulting IHPIs number for any specific method, to compare different parameters sets between each other.

Exemplary results containing ten best, balanced, DNCC with improved stealthiness are presented in the Tab. 5. It must be observed that for the cases enclosed in this table the $\mathrm{CV}$ value is lower than 0.135 . Thus, when compared with the values presented for the careless approach (Tabs. 1 and 2, last column) we can observe that these values are noticeably smaller (i.e., 3-5 times lower). Moreover, it is visible that in comparison to the careless approach the number of IHPIs for each data hiding method is rather balanced and therefore we do not experience noticeably higher values for any single method that could facilitate detection. In fact, we used the same data mining-based detection approach that we utilized for the careless approach and none of the presented configurations 
has been spotted (we omit this table as it will contain only zeros in the summary support part). Note, that the resulting steganographic bandwidth is significantly improved as for these cases it is up to 4.5 times higher when compared to the careless approach and the best covert data rate has been obtained for the following DNCC configuration: $3 ; 2 ; 1$ bit/symbol and 600; 40; 600 symbol frequency for the TTL, HTTP Reorder and TCP options methods, respectively.

\section{CONCLUSIONS}

In this paper we have investigated how distributed network covert channels which combine many data hiding techniques can be formed in order to ensure increased stealthiness. We showed that when the DNCC configuration is selected in a careless manner then it can be often easily detectable as some information hiding methods maybe be used excessively compromising the whole setup. On the other hand, the better approach is to balance the utilization of each of the covert techniques and limit the overall steganographic bandwidth which greatly improves the resulting undetectability. Our future work will be devoted to further analyze the capabilities and potential of the distributed network covert channels and investigate efficient ways to detect them.

\section{ACKNOWLEDGMENTS}

This material is based upon work supported by the Air Force Office of Scientific Research under award number FA9550-17-1-0254. The supported project is named CoCoDe - Covert Communication Detection. Any opinions, finding, and conclusions or recommendations expressed in this material are those of the author(s) and do not necessarily reflect the views of the United States Air Force.

\section{REFERENCES}

[1] P. Backs, S. Wendzel, and J. Keller. 2012. Dynamic routing in covert channel overlays based on control protocols. In 2012 International Conference for Internet Technology and Secured Transactions. 32-39.

[2] K. Cabaj, L. Caviglione, W. Mazurczyk, S. Wendzel, A. Woodward, and S. Zander. 2018. The New Threats of Information Hiding: The Road Ahead. IT Professional 20, 3 (May 2018), 31-39. https://doi.org/10.1109/MITP.2018.032501746

[3] Krzysztof Cabaj, Wojciech Mazurczyk, Piotr Nowakowski, and Piotr Żórawski. 2018. Towards Distributed Network Covert Channels Detection Using Data Mining-based Approach. In Proceedings of the 13th International Conference on Availability, Reliability and Security (ARES 2018). ACM, New York, NY, USA Article 12, 10 pages. https://doi.org/10.1145/3230833.3233264

[4] S. Cabuk, C. E. Brodley, and C. Shields. 2004. IP Covert Timing Channels: Design and Detection. In Proc. 11th ACM conference on Computer and Communications Security. ACM, 178-187.

[5] L. Caviglione, M. Podolski, W. Mazurczyk, and M. Ianigro. 2017. Covert Channels in Personal Cloud Storage Services: The Case of Dropbox. IEEE Transactions on Industrial Informatics 13, 4 (Aug 2017), 1921-1931. https://doi.org/10.1109/TII 2016.2627503

[6] Luca Caviglione, Steffen Wendzel, and Wojciech Mazurczyk. 2017. The future of digital forensics: Challenges and the road ahead. IEEE Security \& Privacy 15, 6 (2017), 12-17.

[7] W. Mazurczyk and L. Caviglione. 2015. Information Hiding as a Challenge for Malware Detection. IEEE Security Privacy 13, 2 (Mar 2015), 89-93. https: //doi.org/10.1109/MSP.2015.33

[8] Wojciech Mazurczyk, Steffen Wendzel, and Krzysztof Cabaj. 2018. Towards Deriving Insights into Data Hiding Methods Using Pattern-based Approach. In Proceedings of the 13th International Conference on Availability, Reliability and Security (ARES 2018). ACM, New York, NY, USA, Article 10, 10 pages. https //doi.org $/ 10.1145 / 3230833.3233261$

[9] W. Mazurczyk, S. Wendzel, S. Zander, A. Houmansadr, and K. Szczypiorski. 2016. Information Hiding in Communication Networks: Fundamentals, Mechanisms, Applications, and Countermeasures. Wiley-IEEE.

[10] Aleksandra Mileva and Boris Panajotov. 2014. Covert channels in TCP/IP protocol stack - extended version-. Central European Journal of Computer Science 4, 2 (01 Jun 2014), 45-66. https://doi.org/10.2478/s13537-014-0205-6
[11] Shishir Nagaraja, Amir Houmansadr, Pratch Piyawongwisal, Vijit Singh, Pragya Agarwal, and Nikita Borisov. 2011. Stegobot: A Covert Social Network Botnet. In Proceedings of the 13th International Conference on Information Hiding (IH'11). Springer-Verlag, Berlin, Heidelberg, 299-313. http://dl.acm.org/citation.cfm?id= 2042445.2042473

[12] Krzysztof Szczypiorski, Igor Margasiński, Wojciech Mazurczyk, Krzysztof Cabaj, and Paweł Radziszewski. 2008. TrustMAS: Trusted Communication Platform for Multi-Agent Systems. In On the Move to Meaningful Internet Systems: OTM 2008, Robert Meersman and Zahir Tari (Eds.). Springer Berlin Heidelberg, Berlin, Heidelberg, 1019-1035.

[13] Steffen Wendzel, Wojciech Mazurczyk, Luca Caviglione, and Michael Meier. 2014. Hidden and Uncontrolled - On the Emergence of Network Steganographic Threats. In ISSE 2014 Securing Electronic Business Processes, Helmut Reimer, Norbert Pohlmann, and Wolfgang Schneider (Eds.). Springer Fachmedien Wiesbaden, Wiesbaden, 123-133.

[14] S. Wendzel, S. Zander, B. Fechner, and C. Herdin. 2015. Pattern-based Survey and Categorization of Network Covert Channel Techniques. Computing Surveys (CSUR) 47, 3 (2015).

[15] S. Zander, G. Armitage, and P. Branch. 2007. A survey of covert channels and countermeasures in computer network protocols. Comm. Surveys and Tutorials 9, 3 (2007), 44-57.

[16] Elżbieta Zielińska, Wojciech Mazurczyk, and Krzysztof Szczypiorski. 2014. Trends in Steganography. Commun. ACM 57, 3 (March 2014), 86-95. https://doi.org/10. $1145 / 2566590.2566610$ 